\title{
A Study on Performance Improvement to Use Dummy Elements on A Monopole Array-assisted Doppler Spread Compensator for
} A Digital Terrestrial Television Broadcasting Receiver

\author{
Young-cheol Yu* Regular Member
}

\begin{abstract}
This paper proposes an array antenna assisted Doppler spread compensator with dummy elements which are placed on either end of a monopole array for a digital terrestrial television broadcasting (DTTB) receiver. An array antenna assisted Doppler spread compensator, proposed previously, has a major drawback in performance degradation owing to mutual coupling effect among array elements. In order to solve the mutual coupling problem, dummy elements, placed on both sides of the monopole array mitigate performance degradation of a Doppler spread compensator arising from the mutual coupling among monopole array elements. Computer simulation results show that the dummy elements can reduce this performance degradation as well as expand the operating bandwidth of a Doppler spread compensator.
\end{abstract}

Key Words : Doppler spread compensator, Mutual coupling, Digital Terrestrial TV, Monopole arrays, OFDM

\section{I . Introduction}

Recently, digital terrestrial television broadcasting (DTTB) has begun in many parts of the world. Among them, the European digital video broadcasting for terrestrial (DVB-T) ${ }^{[1]}$ and the Japanese integrated services digital broadcasting for terrestrial (ISDB-T) ${ }^{[2]}$ employ orthogonal frequency multiplexing division (OFDM) for their transmission schemes. The Korean digital multimedia broadcasting (DMB $)^{[3]}$ and China mobile multimedia broadcasting $(\mathrm{CMMB})^{[4]}$ also employ OFDM. OFDM is capable of high-speed digital transmission in a time-dispersive multi-path channel, and is also robust against frequency selective fading by multi-path delay spreading because of narrow bandwidth between the sub-carriers. Therefore, its hardware complexity is significantly reduced in comparison to a single carrier system a with time-domain equalizer $^{[5]}$. The OFDM system is also capable of single-frequency networks (SFN), which is especially effective in improving frequency utilization efficiency of a national DTTB network.

On the other hand, due to the narrow bandwidth between the sub-carriers, OFDM is sensitive to inter-channel interference (ICI). ICI is caused by Doppler spread, where several incoming waves affect the different Doppler shift, as well as by frequency offset between the transmitter and receiver.

In this paper, we consider Mode3 of the Japanese DTTB system ISDB-T, which has 5617 sub-carriers and $1 \mathrm{kHz}$ of frequency spacing among sub-carriers. The maximum Doppler shift frequency reaches $70 \mathrm{~Hz}$ when the carrier frequency is $770 \mathrm{MHz}$ and the receiver moves at $100 \mathrm{~km} / \mathrm{h}$. This implies that the bit error rate (BER) performance is severely degraded by the

* LG전자 Mobile Communications 연구소(youngc.yu@gmail.com)

논문번호 : KICS2011-10-476, 접수일자 : 2012년 10월 17일, 최종논문접수일자 : 2012년 5월 16일 
논문/ A study on Performance Improvement to use Dummy elements on a Monopole Array-assisted Doppler Spread Compensator for a Digital Terrestrial Television Broadcasting Receiver

Doppler spread effect.

To solve this problem, a linear array antenna-assisted Doppler spread compensator has been proposed by Okada et al. ${ }^{[6-8]}$. The proposed Doppler spread compensator was composed of a linear array antenna and a space domain interpolator. The space domain interpolator estimates the received signals at a fixed position with respect to the ground by using the received signals from each array element. Consequently, it could reduce BER performance degradation caused by the Doppler spread effect.

However, the mutual coupling effect changes the radiation patterns of each array element. The space domain interpolator is difficult to estimate the received signals at a fixed position with respect to the ground because the radiation patterns from each array element are different. Therefore, the performance of a Doppler spread compensator deteriorates due to the mutual coupling effect.

To reduce the mutual coupling effect, the proposed Doppler spread compensator has employed a mutual coupling canceller ${ }^{[7],[8]}$, which calculates a mutual impedance matrix normalized by the load impedance. Hence, the mutual coupling canceller is sensitive to changes of carrier frequency. Moreover, its operating bandwidth is narrow compared with the television frequency $\operatorname{band}^{[7],}{ }^{[8]}$. For reference, the carrier frequency of ISDB-T is from $470 \mathrm{MHz}$ to $770 \mathrm{MHz}$, and its bandwidth is $300 \mathrm{MHz}$.

The use of dummy elements for reducing mutual coupling effect is already proposed ${ }^{[9],}{ }^{[10]}$. In [9], dummy elements are terminated with matched loads on each side of array to provide a similar environment for all the inner array elements. This method is simple way to reduce mutual coupling effect. However, it needs more space to set the arrays and more elements.

In this paper, we use dummy elements, which are attached on both sides of the monopole array and terminated with loads. The mutual coupling effect between main elements is decreased due to coupling between dummy element and main element. Dummy elements can reduce the difference of the radiation pattern between each element of the monopole array. Therefore, our proposed scheme could improve the performance of the Doppler spread compensator.

The rest of the paper is organized as follows. Section II describes the proposed Doppler spread compensator; Section III shows computer simulation results; and Section IV summarizes and makes concluding remarks.

\section{Proposed Doppler Spread Compensator}

Fig. 1 illustrates the proposed Doppler spread compensator. It is composed of two dummy elements, which are placed on both sides of a monopole array. The antenna spacing between each element is $\mathrm{d}$ ( $\lambda$ is $0.6[\mathrm{~m}]$ when carrier frequency is $500 \mathrm{MHz}$ )

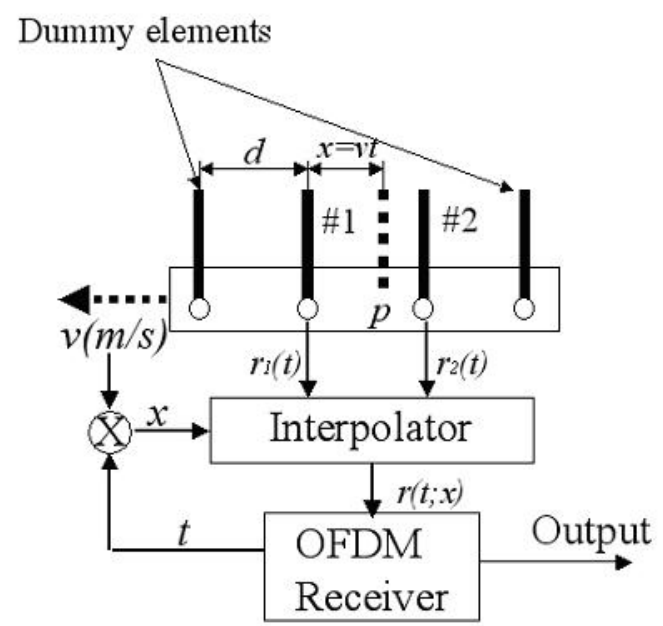

Fig. 1. Block diagram of the Proposed Doppler Spread Compensator

The space domain interpolator estimates a virtual reception point $\mathrm{p}$, which is $\mathrm{x}=\mathrm{vt}$ apart from the first array element. Since $p$ is stationary with respect to the ground, it can compensate for Doppler spread. To calculate $\mathrm{p}$, the space domain interpolator requires vehicle speed information. In the following, we assume perfect knowledge of vehicle speed information. 
The received signal $\mathbf{r}(t)=\left[r_{1} r_{2}\right]^{t}$ is applied to the space domain interpolator to estimate the received signal at position $\mathrm{x}$,

$$
\tilde{r}(t ; x)=\mathrm{w}^{H}(x) \cdot \mathrm{r}(t)
$$

where $\mathrm{w}(x)$ is a weight vector. The derivation of the weight vector is given in the Appendix A.

The output signal of the space domain interpolator is then applied to the OFDM receiver to demodulate the signal.

From Fig. 2, dummy elements terminated with ground. The incoming waves $a_{1}$ and $a_{2}$, which are inputted to \#1 and \#2 elements, couple with each array element. The coupling signals $c_{1}$ and $c_{2}$, which couple between the dummy elements and the monopole array, are eliminated by dummy elements. Therefore, the mutual coupling effect between \#1 and \#2 elements, $d_{1}$ and $d_{2}$, can be reduced.

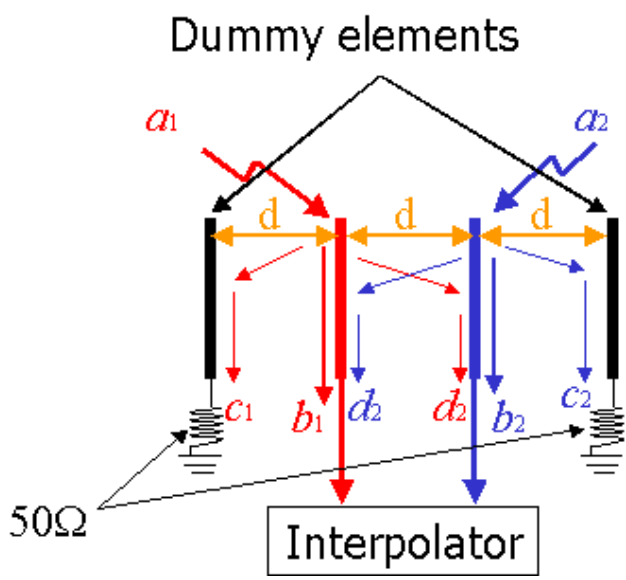

Fig. 2. Effect of Dummy Elements

According to reference ${ }^{[9],[11]}$, mutual coupling affects the radiation pattern. The radiation pattern of an element in an array environment differs from the one in an isolated situation ${ }^{[11]}$.

Dummy elements mitigate performance degradation due to the mutual coupling effect, because they can reduce the difference of the radiation pattern between each element of the monopole array.

\section{Computer Simulation Results}

To confirm the effectiveness of our proposed scheme, we carried out computer simulations. First, we analyzed complex value directivity of the monopole array using the moment method based on an antenna simulation tool, numerical electromagnetic code (NEC-2) ${ }^{[12]}$. The directivity patterns were applied to the fading simulator based on references [13], [14].

In the simulator, the amplitude and phase of each offset oscillator is adjusted according to the directivity.

Table 1. Antenna Simulation Parameters

\begin{tabular}{|l|l|}
\hline Frequency & $500 \mathrm{MHz}$ \\
\hline Load Impedance & $50 \Omega$ \\
\hline Source & Incident Plane Wave \\
\hline Antenna Length & $0.25 \lambda$ \\
\hline
\end{tabular}

Table 1 and Table 2 show antenna simulation parameters and computer simulation parameters, respectively.

In the computer simulation, we supposed Mode3 of ISDB-T standard, without error correction.

Table 2. Computer Simulation Parameters

\begin{tabular}{|c|c|}
\hline \multicolumn{2}{|l|}{ Transmission Parameters } \\
\hline Bandwidth & $5.572 \mathrm{MHz}$ \\
\hline Carrier Spacing & $0.992 \mathrm{kHz}$ \\
\hline FFT Size & 8192 \\
\hline Number of Carriers & 5617 \\
\hline $\begin{array}{l}\text { Number of Data } \\
\text { Carriers }\end{array}$ & 4992 \\
\hline Carrier Modulation & 64 QAM \\
\hline $\begin{array}{ll}\text { Effective } & \text { Symbol } \\
\text { Duration } & \\
\end{array}$ & $1.008 \mathrm{~ms}$ \\
\hline Guard Interval & $128 \mu s(1 / 8)$ \\
\hline \multicolumn{2}{|l|}{ Propagation Parameters } \\
\hline Model & $\begin{array}{l}\text { Equal gain two-ray } \\
\text { Rayleigh fading }{ }^{[13]} \\
\text { GSM Delay Profile }{ }^{[14]} \\
\text { (Hilly terrain 6-ray and } \\
\text { 12-ray) }\end{array}$ \\
\hline Direction of Arrival & Uniform distribution \\
\hline
\end{tabular}


논문 / A study on Performance Improvement to use Dummy elements on a Monopole Array-assisted Doppler Spread Compensator for a Digital Terrestrial Television Broadcasting Receiver

According to Appendix B, the optimum antenna spacing for the ideal monopole array is $0.075 \lambda$, while the optimum antenna spacing for 2,4 and 6-element monopole array with mutual coupling effect are $0.425 \lambda, 0.25 \lambda$ and $0.3 \lambda$, respectively at $E_{b} / N_{0}=35 \mathrm{~dB} \quad$ and $f_{d} T_{s}=0.1 \quad\left(f_{d} T_{s}\right.$ is the maximum Doppler shift frequency normalized by the effective symbol duration).

In the following simulation, we have made use of the optimum antenna spacing.

First, we investigated the radiation patterns of each main element. According to the reference [9], [11], radiation patterns of each main element are disturbed and the main elements will no longer be identical due to mutual coupling. Therefore, we can measure that the mutual coupling effect by comparing the radiation pattern of main elements. The smaller the radiation pattern difference is the less the degradation due to mutual coupling.

The radiation patterns are shown in Figure 3. The difference between the radiation patterns of 2-element monopole array is lager than that of 4 and 6-element monopole arrays. The difference between the radiation patterns of 6-element monopole is smaller than other monopole arrays. It means that the mutual coupling effect of 6-element monopole array is less than other monopole arrays.

In the following, the computer simulation results show the proposed scheme is capable of improving BER performance by reducing mutual coupling effect.

Fig. 4 shows BER performance against $f_{d} T_{s}$, when $E_{b} / N_{0}$ is $35 \mathrm{~dB}$ and is using the optimum antenna spacing. 'w/o compensator' means it does not employ Doppler spread compensator. The ideal monopole array, which has 2 elements and in which mutual coupling effect is not considered, gives better BER performance than a 6-element monopole array.

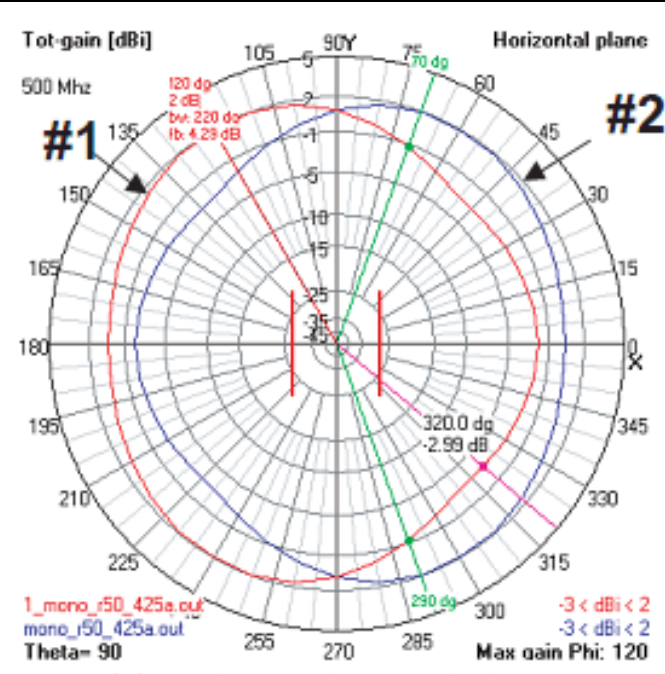

(a) 2-Element Monopole Array

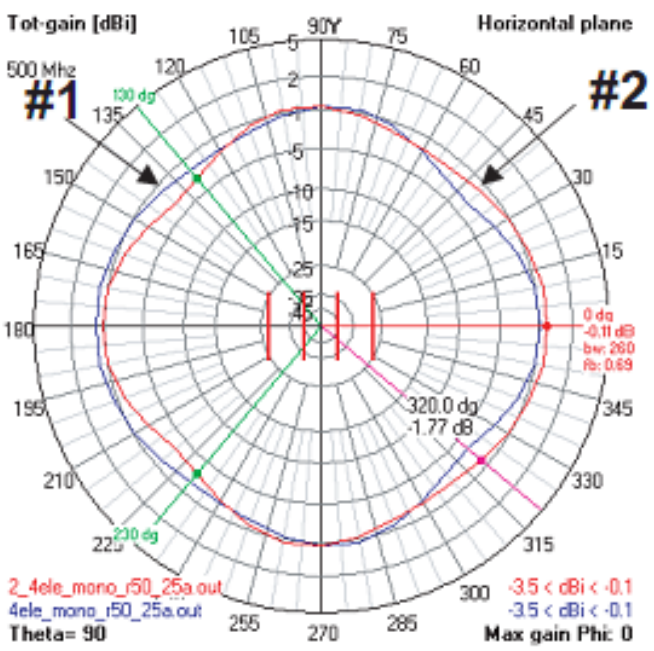

(b) 4-Element Monopole Array

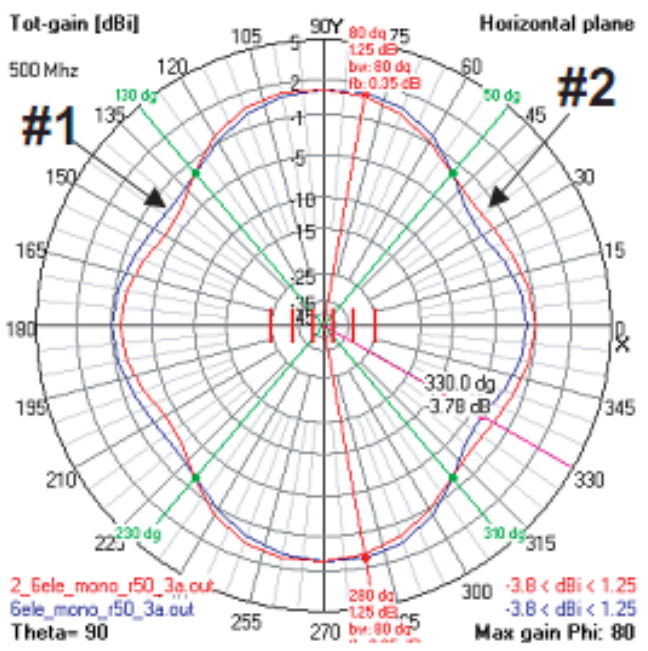

(c) 6-Element Monopole Array

Fig. 3. Radiation Patterns $\left(f_{c}=500 \mathrm{MHz}\right.$, Antenna Length $\mathrm{L}=0.15[\mathrm{~m}]$ ) 


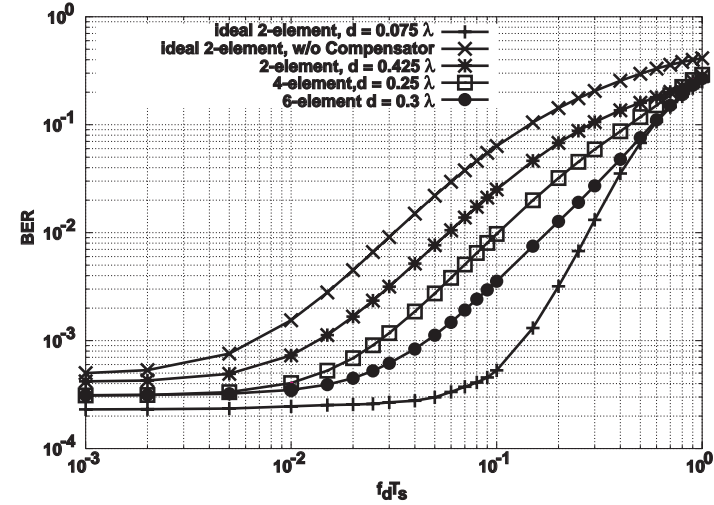

Fig. 4. BER Performance against $f_{d} T_{s}\left(E_{b} / N_{0}\right.$ $=35 \mathrm{~dB}$ )

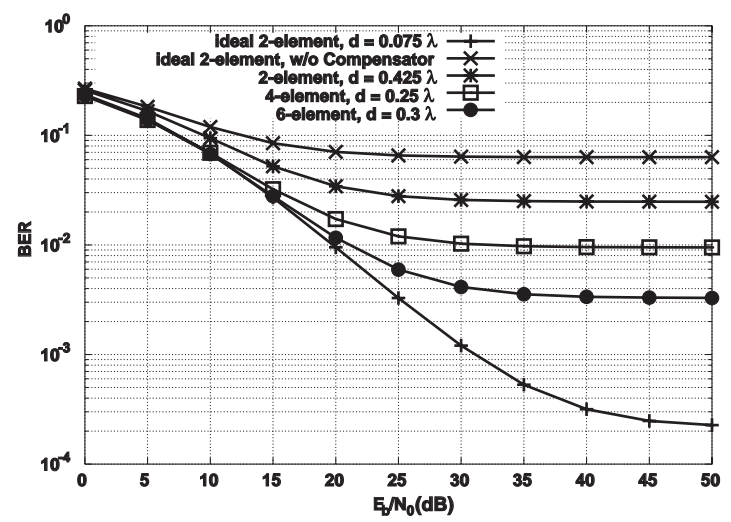

Fig. 5. BER Performance against $E_{b} / N_{0}\left(f_{d} T_{s}\right.$ $=0.1)$

Fig. 5 represents BER performance versus when $f_{d} T_{s}$ is 0.1 and the optimum antenna spacing is used. The 2-element monopole array has a better BER performance than an ideal monopole array without a Doppler spread compensator. The 6-element monopole array gives better BER performance than a 2-element or 4-element monopole array.

The ideal monopole array, which has 2 elements and in which the mutual coupling effect is not considered, gives better BER performance than the 6-element monopole array.

According to Figures 4 and 5, 6-element monopole array has better BER performance than other monopole arrays (2-element, 4-element) which consider the mutual coupling effect. We know that dummy elements effectively reduce the mutual coupling effect. However, the proposed scheme takes much space due to the dummy elements.

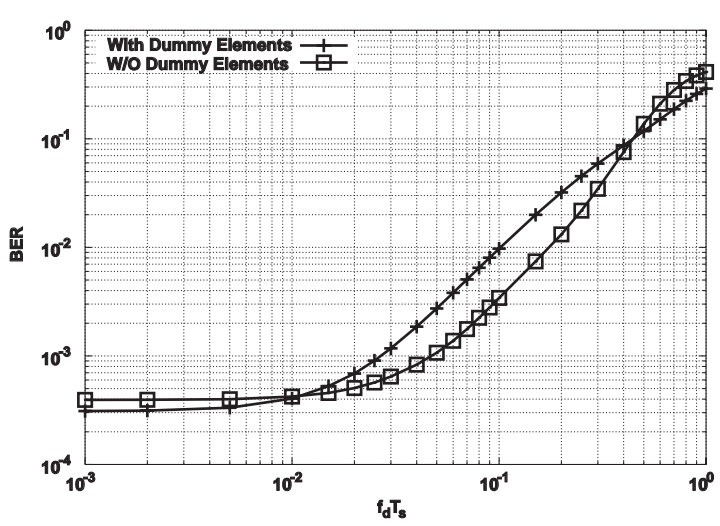

(a) BER Performance against $f_{d} T_{s}\left(E_{b} / N_{0}=35 \mathrm{~dB}\right)$

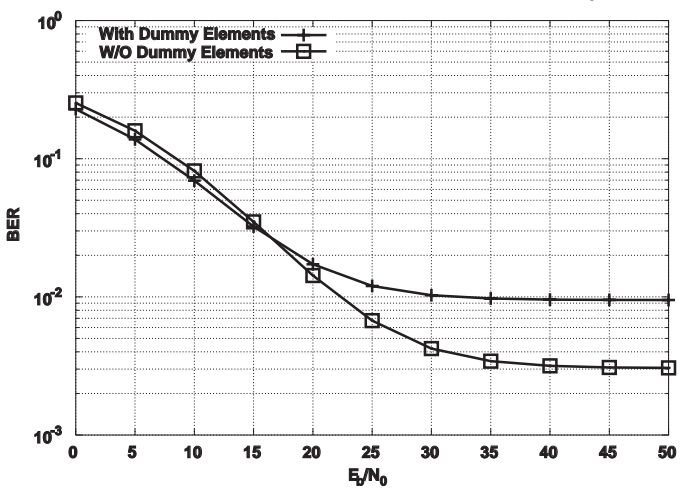

(b) BER Performance against $E_{b} / N_{0} \quad\left(f_{d} T_{s}\right.$ $=0.1$ )

Fig. 6. BER Performance of 4-element with or without Dummy elements

In Figure 6, it compares BER performance 4-element monopole array with dummy elements and without dummy elements. All the array elements are used for Doppler spread compensator in the case of 4-element monopole array without dummy elements.

From Fig. 6, 4-element monopole array without dummy elements has better BER performance than 4-element monopole array with dummy elements. However, there is little difference in BER performance between 4-element monopole array with dummy elements and without dummy elements.

Fig. 7 shows BER performance against carrier frequency, which is from $400 \mathrm{MHz}$ to $770 \mathrm{MHz}$. '(x)' indicates the monopole array antenna is designed for $500 \mathrm{MHz}$, while '(y)' indicates the monopole array is designed for $700 \mathrm{MHz}$. The 
논문 / A study on Performance Improvement to use Dummy elements on a Monopole Array-assisted Doppler Spread Compensator for a Digital Terrestrial Television Broadcasting Receiver

antenna spacing for $700 \mathrm{MHz}$ is obtained by optimization. The optimization result is given in Appendix B.

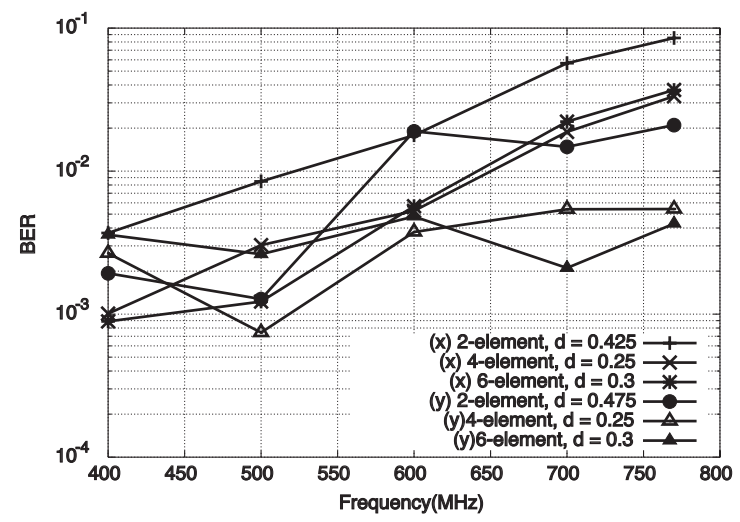

(a) Vehicle Speed, v $=100 \mathrm{~km} / \mathrm{h}$

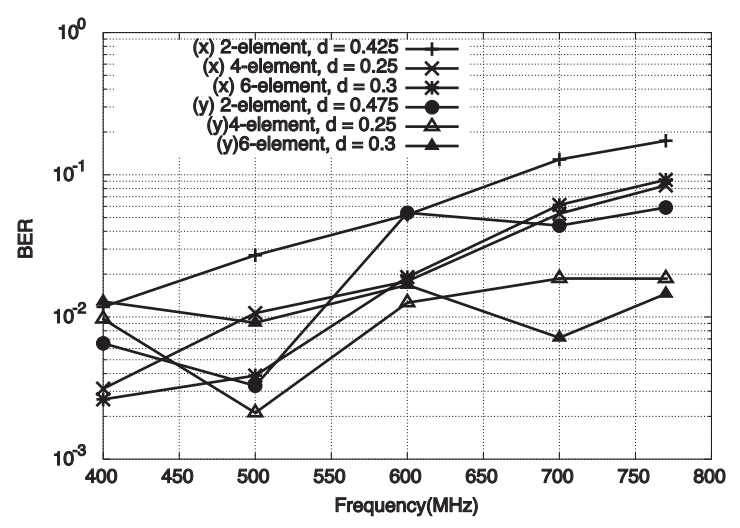

(b) Vehicle Speed, v $=200 \mathrm{~km} / \mathrm{h}$

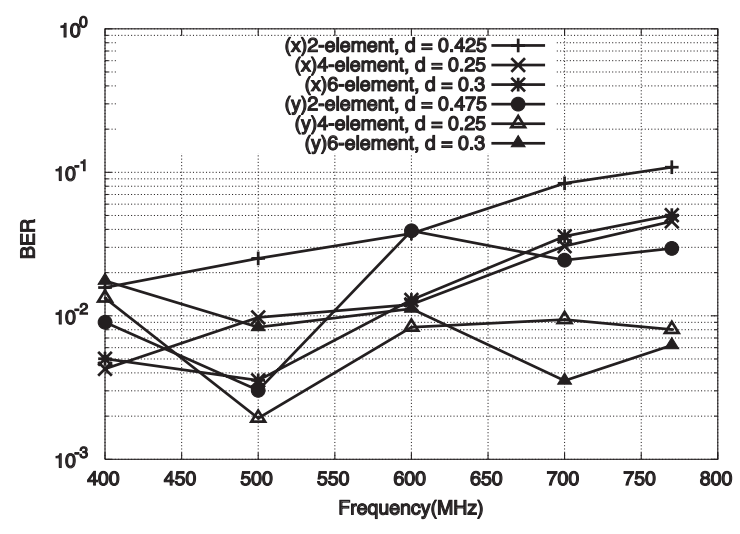

(c) $f_{d} T_{s}=0.1$

Fig. 7. BER Performance against Carrier Frequency $\left(E_{b} / N_{0}=35 \mathrm{~dB}\right)$

Fig. 7(a), 7(b) represent BER performance versus carrier frequency, when $E_{b} / N_{0}$ is $35 \mathrm{~dB}$ and the vehicle speeds are $100 \mathrm{~km} / \mathrm{h}$ and $200 \mathrm{~km} / \mathrm{h}$, respectively. In Fig.7(a) and 7(b), the vehicle speed is fixed as $100 \mathrm{~km} / \mathrm{h}$ and $200 \mathrm{~km} / \mathrm{h}$ however, $f_{d} T_{s}$ is changed because the carrier frequency is changed. On the other hand, in Fig. 7(c), the maximum Doppler shift frequency normalized by the effective symbol duration,, is fixed as 0.1 regardless of the carrier frequency. The BER performance is deteriorated when vehicle speed is high.

From Fig. 7, BER performance of the monopole array which is designed for $500 \mathrm{MHz}$ has deteriorated when carrier frequency is higher than 500MHz. Meanwhile, the monopole array which is designed for $700 \mathrm{MHz}$ has better BER performance than $500 \mathrm{MHz}$.

On the other hand, 2-element monopole array for $700 \mathrm{MHz}$ gives better BER performance than 6-element monopole array for $700 \mathrm{MHz}$ when the carrier frequency is $500 \mathrm{MHz}$. According to Fig. 8, the radiation pattern difference of 2-element monopole array and 4-element monopole array is smaller than that for 6-element monopole array. Therefore, 2-element monopole array designed for $700 \mathrm{MHz}$ gives better BER performance than 6-element monopole array for $700 \mathrm{MHz}$, when BER is measured at the carrier frequency of $500 \mathrm{MHz}$.

The Operating bandwidth is the frequency region that the degradation due to mutual coupling is small enough for receiving the ISDB-T signal. As the required BER, we assume $2 \times 10^{-2}$ before FEC when BER before inner code decoding is below $2 \times 10^{-2}$, quasi error free(QEF) rate of $10^{-11}$ can be obtained after forward error correction(FEC) ${ }^{[15]}$. The receiver satisfies the required BER of $2 \times 10^{-2}$ in the operating bandwidth region.

The previously proposed method used mutual coupling canceller ${ }^{[7], ~[8], ~ h o w e v e r, ~ i t s ~ o p e r a t i n g ~}$ bandwidth is $30 \mathrm{MHz}$, which the total frequency band assigned for ISDB-T is $300 \mathrm{MHz}$. It is difficult to design the mutual coupling canceller algorithm, which can compensate for throughout 
the ISDB-T band. On the other hand, the operating bandwidth of the monopole array with dummy elements for $700 \mathrm{MHz}$ is $370 \mathrm{MHz}$. It is sufficient to cover ISDB-T bandwidth. Therefore, the proposed scheme provides wide operating bandwidth in order to add dummy elements on both sides of main elements.

Meanwhile, the monopole array with dummy elements which is designed for $700 \mathrm{MHz}$ has wide operating bandwidth irrespective of the vehicle speed.

Fig. 9 shows BER performance of a monopole array versus various delay profiles. The delay profiles used the equal gain 2-ray Jakes model ${ }^{[13]}$ and the hilly terrain 6-tap and 12-tap model of the global system for mobile communication $(\mathrm{GSM})^{[14]}$. Figure 9(a) and Figure 9(b) show BER performance versus $f_{d} T_{s}$, when $E_{b} / N_{0}$ is $35 \mathrm{~dB}$, and BER performance against $E_{b} / N_{0}$, when $f_{d} T_{s}$ is0.1,respectively.

According to Fig. 9, although, the two-ray Rayleigh fading model is more severe environment than 6-ray and 12-ray models. The reason is that delay of the two-ray fading model is within a guard interval. Also, our proposed dummy elements attached on both sides of a monopole array give good BER performance in various mobile environments.

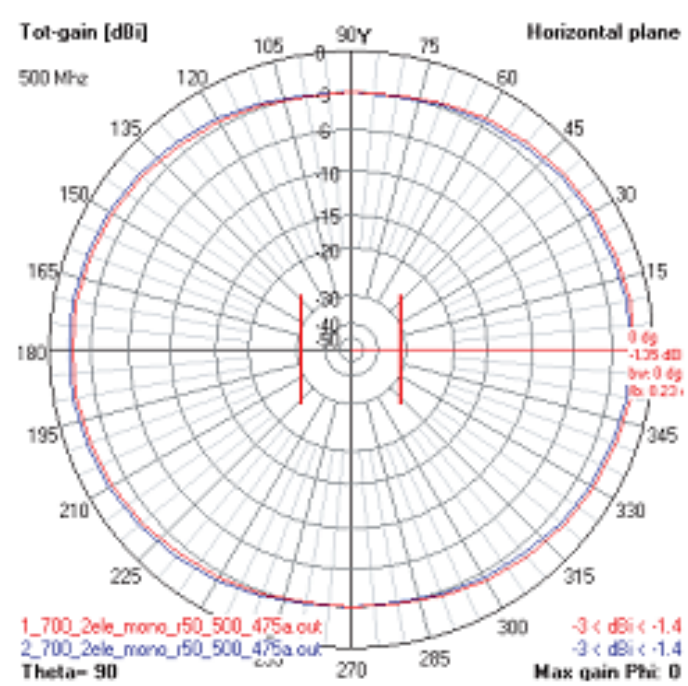

(a) 2-Element Monopole Array

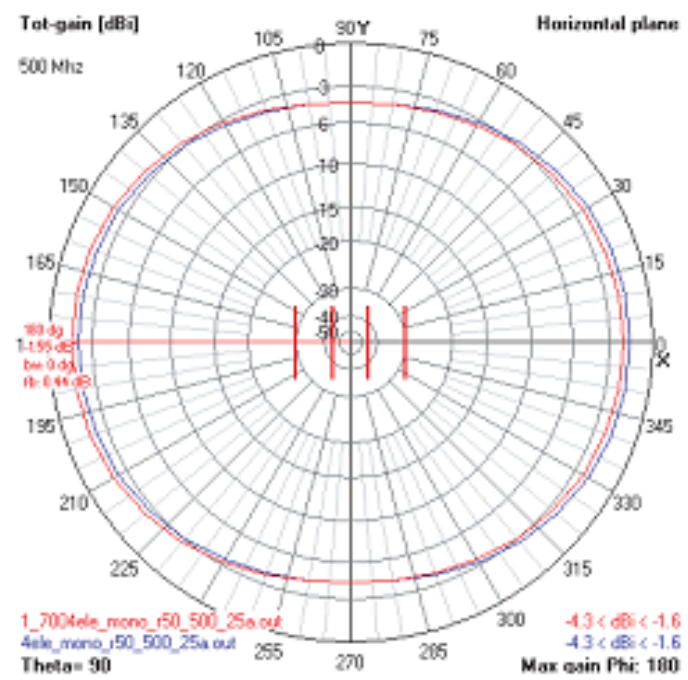

(b) 4-Element Monopole Array

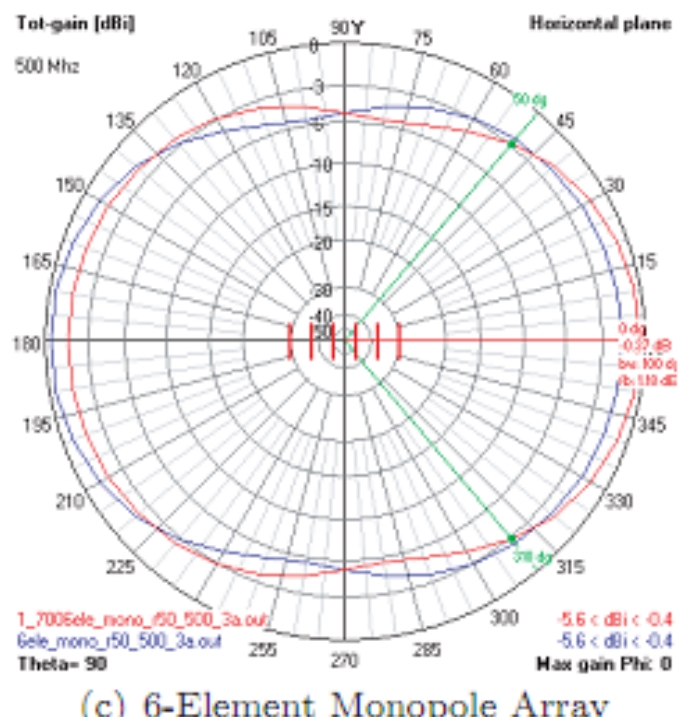

Fig. 8. Radiation Patterns $\left(f_{c}=500 \mathrm{MHz}\right.$, Antenna Length $\mathrm{L}=0.107[\mathrm{~m}])$ 
논문 / A study on Performance Improvement to use Dummy elements on a Monopole Array-assisted Doppler Spread Compensator for a Digital Terrestrial Television Broadcasting Receiver

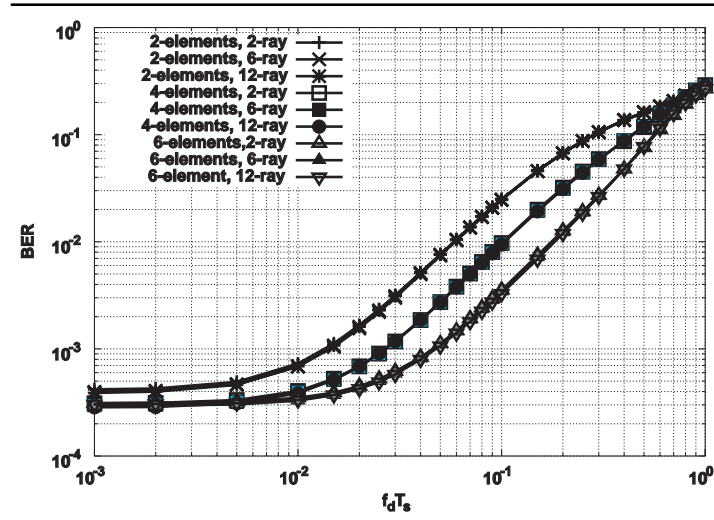

(a) BER Performance against $f_{d} T_{s}\left(E_{b} / N_{0}=35 \mathrm{~dB}\right)$

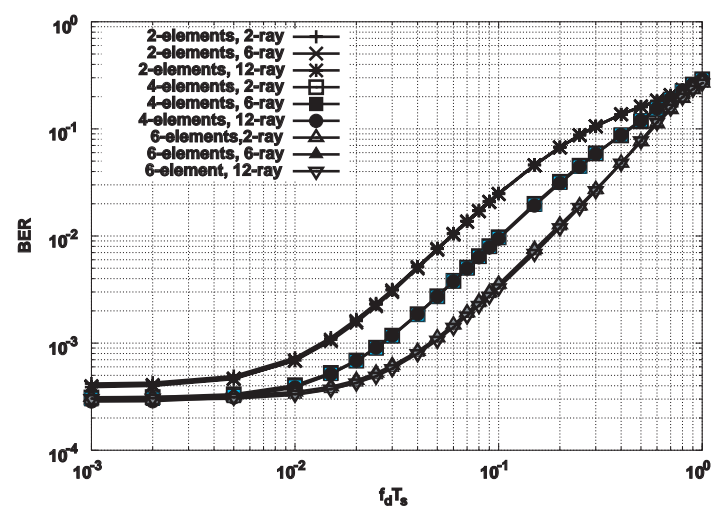

(b) BER Performance against $E_{b} / N_{0}\left(f_{d} T_{s}=0.1\right)$

Fig. 9. BER Performance against Various Fading Models

\section{Conclusion}

In this paper, we have proposed dummy elements attached on both sides of a monopole array, which have an assisted Doppler spread compensator and a reduced mutual coupling effect. Also, they expand the operating bandwidth of a Doppler spread compensator.

The optimum antenna spacing was evaluated by computer simulation, and we then measured BER performance of our proposed dummy elements attached on both sides of a monopole array versus Doppler shift frequency, $E_{b} / N_{0}$ carrier frequency and various mobile environments. Also, the radiation pattern was evaluated by the antenna simulation tool NEC-2.

From antenna simulation results, we found that the difference in radiation patterns of each element becomes smaller if the number of dummy elements is increased. Therefore, the difference in radiation patterns of each element of a 6-element monopole array is smaller than other monopole arrays (2-element and 4-element).

According to computer simulation results, an ideal monopole array, which does not assume a mutual coupling effect, with a Doppler spread compensator has better BER performance than other monopole arrays (2-element, 4-element, 6-element). However, the BER performance of the 2-element monopole array, which assumes mutual coupling effect, has deteriorated. If the number of dummy elements is increased, BER performance is improved.

Consequently, the proposed dummy elements attached on both sides of a monopole array effectively mitigate performance degradation due to the mutual coupling effect. They also expand the operating bandwidth of a Doppler spread compensator as well as having good BER performance in various mobile environments.

\section{Appendix A}

The space domain interpolator uses a minimum mean square error (MMSE) algorithm. Let us suppose that the two-dimensional received signal vector $r(t)$ is:

$$
\mathbf{r}(t)=\left[r_{1}(t) r_{2}(t]^{T}\right.
$$

where $r_{1}(t), r_{2}(t)$ are the received signals from the \#1 and \#2 array elements at time t, respectively. The output of the space domain interpolator is given by:

$$
\tilde{r}(x, t)=\mathrm{w}^{T}(x) \cdot \mathrm{r}(t)
$$

where $\tilde{r}(x, t)$ is the estimated signal at position $\mathrm{x}$ and $\mathrm{w}(x)$ is the weight vector, which uses the MMSE algorithm.

The weight vector, $\mathrm{w}(x)$, is given by:

$$
\widetilde{w}(x)=\mathrm{R}^{-1} \cdot \mathrm{b}(x)
$$


where $\mathrm{R}$ denotes the correlation matrix of the received signal vector $\mathrm{r}(t)$ and $\mathrm{b}(x)$ is the cross-correlation vector between the received signal vector and the desired signal $r(x, t)$ and are defined as

$$
\begin{aligned}
& \mathrm{R}=\frac{1}{2}\left[\mathrm{r}(t) \mathrm{r}^{H}(t)\right] \\
& R_{n, k}=\left[J_{0}(2 \pi d(n-k) \lambda)\right]
\end{aligned}
$$

and

$$
\begin{aligned}
& \mathrm{b}(x)=\frac{1}{2} E\left[\mathrm{r}(t) r(x, t)^{*}\right] \\
& b_{k}=\left[J_{0}(2 \pi(k d-x) \lambda)\right]
\end{aligned}
$$

where $\mathrm{k}$ indicates array elements \#1 and \#2 (e.g. $\mathrm{k}=0,1$ ), represents the Hermitian transpose of $x$ and $x^{*}$ is the complex conjugate of $x$. $J_{0}(x)$ is the zero-th order Bessel function of the first kind.

\section{Appendix B}

The optimum antenna spacing, which minimizes BER, is obtained by computer simulation, which uses Jake's fading model ${ }^{[13]}$. That is, there are many incoming paths, where direction of arrivals are uniformly distributed.

Fig. A1(a)-(c) show BER performance versus antenna spacing $\mathrm{d} \lambda$, when $E_{b} / N_{0}$ is $35 \mathrm{~dB}$, carrier frequency is $500 \mathrm{MHz}$ and $f_{d} T_{s}$ is 0.05 , 0.1 and 0.15 , respectively.

According to Fig. A1, the optimum antenna spacing $\mathrm{d} \lambda$ of 2-element monopole array is 0.425 $\lambda$; and 4-element monopole array, which has 2 dummy elements on both sides of monopole array, is $0.25 \lambda$. Also, the optimum antenna spacing of a 6-element monopole array, which has 4 dummy elements on both sides of monopole array, is $0.3 \lambda$. In the case of the ideal monopole array, the optimum antenna spacing $d \lambda$ is changed in accordance with $f_{d} T_{s}$. The optimum antenna spacings of ideal monopole array are
$0.125 \lambda, 0.75 \lambda$ and $0.1 \lambda$ when $f_{d} T_{s}$ is $0.05,0.1$ and 0.15 , respectively. However, the difference in antenna spacing gives little impact on the BER performance.

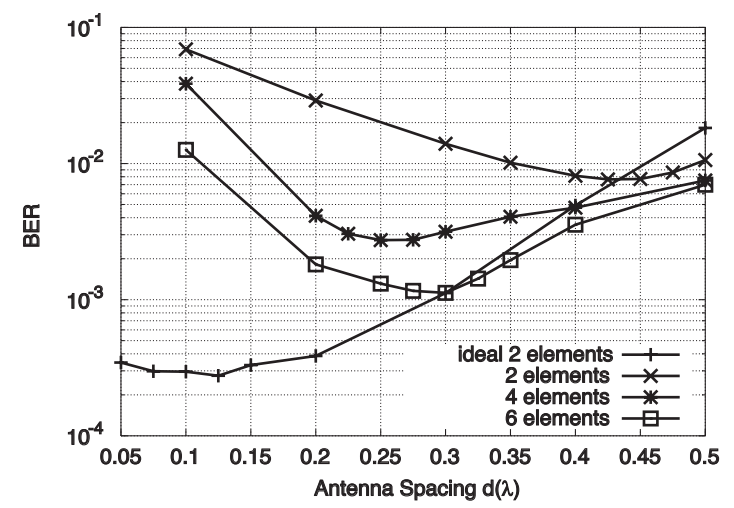

(a) $f_{d} T_{s}=0.05$

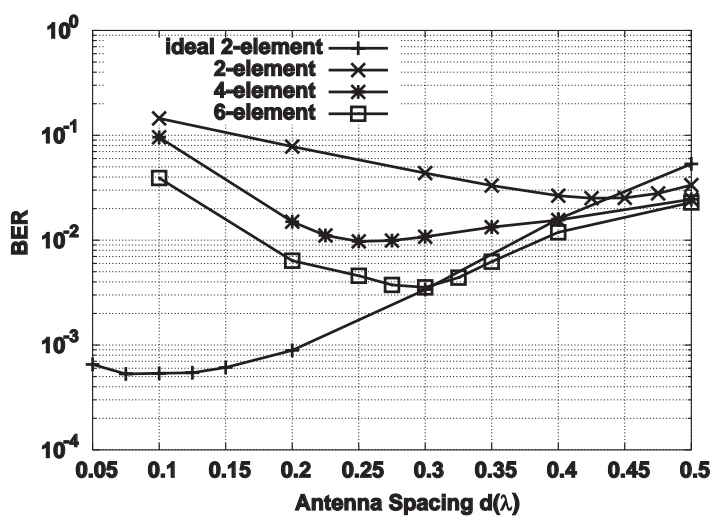

(b) $f_{d} T_{s}=0.1$

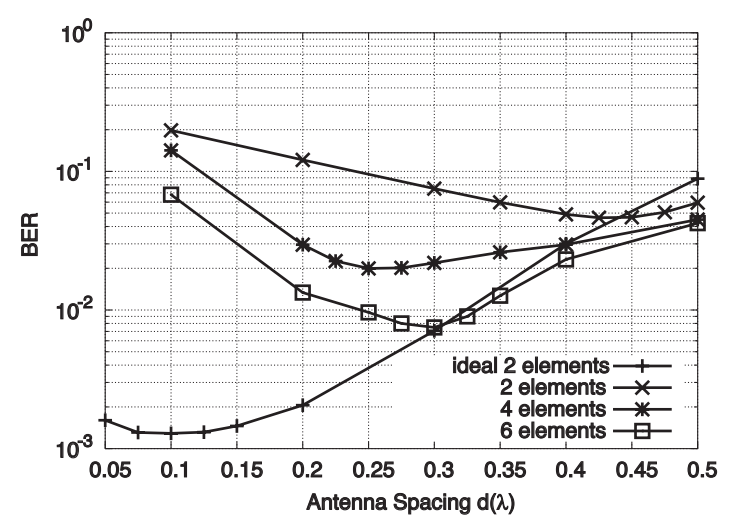

(c) $f_{d} T_{s}=0.15$

Fig. A1. Optimization vs. Antenna Spacing $\left(E_{b} / N_{0}=35 \mathrm{~dB}, f_{c}=500 \mathrm{MHz}\right)$

The optimum antenna spacing with mutual coupling is shown in Fig. A2 when $f_{d} T_{s}$ is 0.1 , 
논문 / A study on Performance Improvement to use Dummy elements on a Monopole Array-assisted Doppler Spread Compensator for a Digital Terrestrial Television Broadcasting Receiver

$E_{b} / N_{0}$ is $35 \mathrm{~dB}$ and carrier frequency is 700MHz. The optimum antenna spacing of 2-element monopole array is $0.475 \lambda$, 4-element monopole array is $0.25 \lambda$ and 6-element monopole array is $0.3 \lambda$, respectively. In this case, $\lambda$ is expressed wavelength at $700 \mathrm{MHz}$ and the value is approximately $0.428 \mathrm{~m}$.

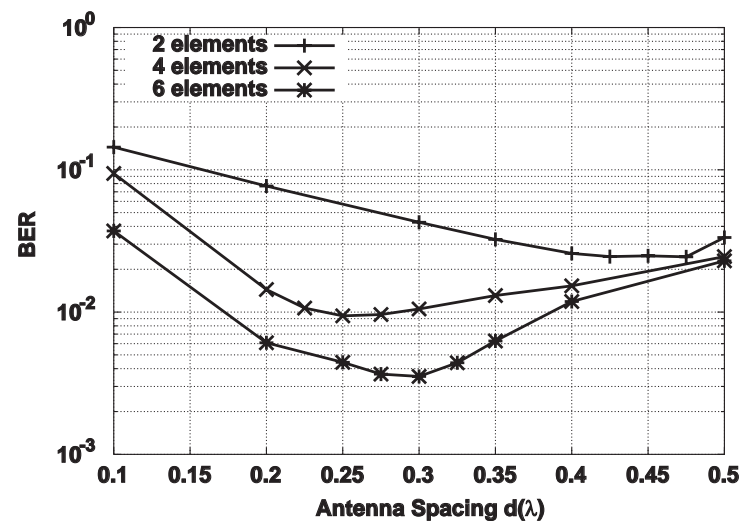

Fig. A2. Optimization vs. Antenna Spacing $\left(f_{d} T_{s}\right.$ $\left.=0.1, E_{b} / N_{0}=35 \mathrm{~dB}, f_{c}=700 \mathrm{MHz}\right)$

According to Fig. A1 and A2, the BER performance became worse even mutual coupling is reduced by extending the antenna spacing. The reason is that the received signals from the array elements should be correlated with each other in order to estimate the received signal at a certain reception point between two elements. Therefore, performance of the Doppler spread compensator has deteriorated if the antenna spacing reaches 0.5 $\lambda$, even if it could reduce mutual coupling effect.

On the other hand, if the antenna spacing is narrow, performance of the Doppler spread compensator has deteriorated due to mutual coupling effect while the correlation between two signals grows. In the case of the ideal monopole array simulation, it does not take into account the effect of mutual coupling but the correlation between array elements is considered.

There is a trade-off between the correlation and mutual coupling. In order to satisfy the both condition, we have to set the antenna spacing to minimize the BER as shown in Fig A1 and A2. The Doppler spread compensator, which uses the optimum antenna spacing, should have good BER performance in fast fading environment. Besides, the proposed scheme prevents the Doppler spread compensator performance degradation due to mutual coupling effect in fast fading environment. Therefore, the optimum antenna spacing has validity in fast fading environment.

\section{Appendix C}

The mutual resistance $R_{21}$ and the mutual reactance $\mathrm{X}_{21}$ of 2-element monopole array can be expressed as in ${ }^{[16]}$ by

$$
\begin{aligned}
\mathrm{R}_{21}= & \frac{15}{\sin ^{2}(\beta L / 2)}\{2(2+\cos \beta L) \operatorname{Ci} \beta d \\
& -4 \cos ^{2} \frac{\beta L}{2}\left[\operatorname{Ci} \frac{\beta}{2}\left(\sqrt{4 d^{2}+L^{2}}-L\right)\right. \\
& \left.+\operatorname{Ci} \frac{\beta}{2}\left(\sqrt{4 d^{2}+L^{2}}+L\right)\right]+\cos \beta L\left[\operatorname{Ci} \beta\left(\sqrt{d^{2}+L^{2}}-L\right)\right. \\
& \left.+\operatorname{Ci} \beta\left(\sqrt{d^{2}+L^{2}}+L\right)\right]+\sin \beta L\left[\operatorname{Si} \beta\left(\sqrt{d^{2}+L^{2}}+L\right)\right. \\
& -\operatorname{Si} \beta\left(\sqrt{d^{2}+L^{2}}-L\right)-2 \operatorname{Si} \frac{\beta}{2}\left(\sqrt{4 d^{2}+L^{2}}+L\right) \\
& \left.\left.+2 \operatorname{Si} \frac{\beta}{2}\left(\sqrt{4 d^{2}+L^{2}}-L\right)\right]\right\}(\Omega),
\end{aligned}
$$

$$
\begin{aligned}
\mathrm{X}_{21}= & \frac{15}{\sin ^{2}(\beta L / 2)}\{-2(2+\cos \beta L) \operatorname{Si} \beta d \\
& +4 \cos ^{2} \frac{\beta L}{2}\left[\operatorname{Si} \frac{\beta}{2}\left(\sqrt{4 d^{2}+L^{2}}-L\right)\right. \\
& \left.+\operatorname{Si} \frac{\beta}{2}\left(\sqrt{4 d^{2}+L^{2}}+L\right)\right]-\cos \beta L\left[\operatorname{Si} \beta\left(\sqrt{d^{2}+L^{2}}-L\right)\right. \\
& \left.+\operatorname{Si} \beta\left(\sqrt{d^{2}+L^{2}}+L\right)\right]+\sin \beta L\left[\operatorname{Ci} \beta\left(\sqrt{d^{2}+L^{2}}+L\right)\right. \\
& -\operatorname{Ci} \beta\left(\sqrt{d^{2}+L^{2}}-L\right)-2 \operatorname{Ci} \frac{\beta}{2}\left(\sqrt{4 d^{2}+L^{2}}+L\right) \\
& \left.\left.+2 \operatorname{Ci} \frac{\beta}{2}\left(\sqrt{4 d^{2}+L^{2}}-L\right)\right]\right\}(\Omega),
\end{aligned}
$$

where $\mathrm{L}$ indicates antenna length, $\mathrm{d}$ means antenna spacing and $\beta$ is $2 \pi / \lambda, \lambda$ being the wavelength of the transmitted carrier frequency. Also, cosine integral and sine integral are given by

$$
\mathrm{Ci}(x)=\gamma+\ln (x)+\int_{0}^{x} \frac{\cos t-1}{t} d t
$$

(A10)

$$
\operatorname{Si}(x)=\int_{0}^{x} \frac{\sin t}{t} d t
$$

where $\gamma$ is Euler's constant 0.577215664 . 
Consequently, the mutual impedance $Z$ is given by

$$
\mathrm{Z}=\mathrm{R}_{21}+j \mathrm{X}_{21}
$$

The mutual impedance of 2-element monopole array is shown in Fig A3, which is obtained by using equation A8, A9 and A12. Fig. A3(a) shows the mutual impedance of 2-element monopole array which is designed for $500 \mathrm{MHz}$ against carrier frequency, where the antenna spacing $\mathrm{d} \lambda$ is $0.425 \lambda$. Also, the mutual impedance of 2-element monopole array which is designed for $700 \mathrm{MHz}$ against carrier frequency is shown in Fig.A3(b), where the antenna spacing $d$ is $0.475 \lambda$.

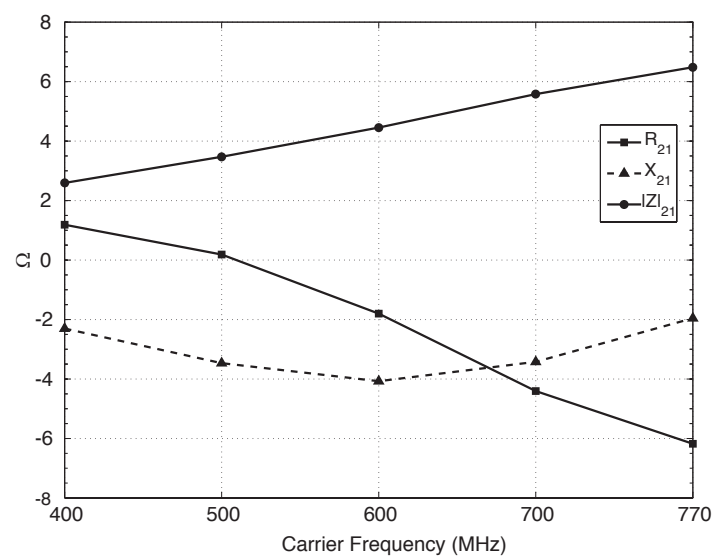

(a) Antenna Length $\mathrm{L}=0.15 \mathrm{~m}$, Antennas Spacing $\mathrm{d}=0.425 \lambda(\lambda=0.6 \mathrm{~m})$

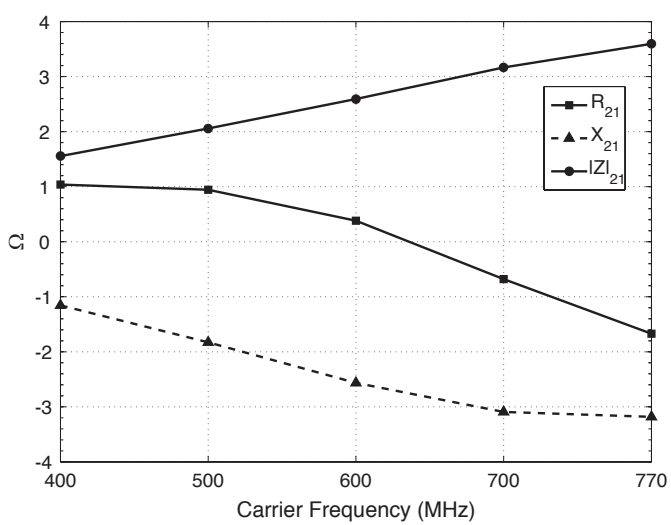

(b) Antenna Length $\mathrm{L}=0.107 \mathrm{~m}$, Antennas Spacing $\mathrm{d}=0.475 \lambda(\lambda=0.428 \mathrm{~m})$

Fig. A3. Mutual Impedance against Carrier Frequency

From Fig. A3, we know that the mutual impedance is increased in accordance with carrier frequency.

\section{References}

[1] Digital Video Broadcasting (DVB) for terrestrial television, ETSI Standard EN 300 744 v.1.4.1, 2001.

[2] Transmission for digital terrestrial television broadcasting, ISDB-T, ARIB standard STD-B31 v.1.5.

[3] Radio Broadcasting Systems: Specification of the video services for VHF Digital Multimedia Broadcasting (DMB) to mobile, portable and fixed receivers, TTA Standard TTAS.KO-07.0026.

[4] Mobile Multimedia Broadcasting Part 1 : Frame Structure, Channel Coding and Modulation for Broadcasting Channel, Chinese Standard of Radio, Film and Television industry. GY/T 220.1-2006, Oct. 2006.

[5] R. V. Nee, R. Prasad, OFDM For Wireless Multimedia Communications, Boston: Artech House Publishers, 2000.

[6] M. Okada and S. Komaki, "Random FM Noise Compensation Scheme for OFDM," Electronics Letters, Vol.36, No.19, pp.1653-1654, September 2000.

[7] M. Okada, H. Takayanagi, H. Yamamoto, "Array Antenna Assisted Doppler Spread Compensator for OFDM," ETT Vol. 13, No. 5, September/October 2002.

[8] M. Okada, H. Takayanagi, H. Yamamoto, "Array antenna assisted Doppler spread compensator for mobile reception of terrestrial television broadcasting," Journal of Institute of Image Information and Television Engineers, vol.56, no.2. pp.237-244, February 2002.

[9] S. Lundgren, "A study of mutual coupling effects on the direction finding performance of ESPRIT with a linear microstrip patch array using the Method of Moments," IEEE Antennas and Propagation Society 
논문/ A study on Performance Improvement to use Dummy elements on a Monopole Array-assisted Doppler Spread Compensator for a Digital Terrestrial Television Broadcasting Receiver

International Symposium, vol.2, pp.1372-1375, July 1996.

[10] B. Lindmark, "Comparison of Mutual Coupling Compensation to Dummy Columns in Adaptive Antenna Systems," IEEE Trans. on Antennas and Propagation, vol.53, no.4, April 2005.

[11] Hubregt J. Visser. Array and Phased Array Antenna Basics, England: Wiley, 2005.

[12] www.nec2.org

[13] William C. Jakes. Microwave Mobile Communications, New York: IEEE Press, 1974.

[14] ETSI GSM recommendation 05.05, Annex C, 1994.

[15] Terrestrial Digital Television Broadcasting, NHK STRL, Broadcast Technology, no.20, Autumn 2004.

[16] John D. Kraus. Antennas, 2nd Edition, USA: McGraw-Hill, 1998. 